European Journal of Mechanics - B/Fluids

November-December 2016, Volume 60, Pages 219-229

http://dx.doi.org/10.1016/i.euromechflu.2016.06.010

http://archimer.ifremer.fr/doc/00344/45503/

(c) 2016 Elsevier Masson SAS. All rights reserved.
Achimer

http://archimer.ifremer.fr

\title{
Analysis of hydrodynamics of a moving trawl codend and its fluttering motions in flume tank
}

\author{
Druault Philippe ${ }^{1,{ }^{*}}$, Germain Gregory ${ }^{2}$
}

${ }^{1}$ Sorbonne Universités, UPMC Univ Paris 06, CNRS, UMR 7190, Institut Jean Le Rond d'Alembert, F75005 Paris, France

${ }^{2}$ IFREMER, Marine Structures Laboratory, 150 Quai Gambetta, 62321 Boulogne-sur-Mer, France

* Corresponding author : Philippe Druault, email address : philippe.druault@upmc.fr gregory.germain@ifremer.fr

\begin{abstract}
:
The coupled dynamics of the codend fluttering motion and its hydrodynamics behavior are investigated based on PIV measurements carried out around a 1/10 scaled model codend. The mean wake flow characteristics are firstly analyzed demonstrating the great effect of the codend motion. The differences in flow contour between fluttering motion and no-fluttering codend are highlighted by analyzing the classical mean characteristics of a body wake flow. In presence of fluttering motion, the classical results are recovered only if one considers the vertical extent of the codends oscillations as a reference length scale. Secondly, the frequency content of codend oscillations is analyzed and it is linked to the physical mechanisms. It is observed that the motion of the codend is mainly of a low-frequency activity and of another component related to the vortex shedding street. It is then demonstrated a complex fluidstructure interaction where the near wake hydrodynamics of the moving codend structure are an intricate interplay between the codend fluttering motion and the shear layer instabilities and large scale vortex shedding. The knowledge of such flow instabilities is of great importance to improve the understanding of the force acting on a trawl and for the implementation of some selected device to drive fish near the codend and then to reduce juvenile by-catch.
\end{abstract}

Keywords : PIV measurements, Fishing net structure, Wake hydrodynamics, Fluttering structure

\section{Introduction}

Trawls funnel-shaped nets are dragged horizontally in the ocean. To optimize their efficiency in terms of catch capability and their impact on the fuel consumption, they must have a high static stability which is difficult because of the great influence of hydrodynamic effects. It is now well known that the turbulent flow developing around fishing nets structures impacts the whole 
drag force of the fishing gear and the selectivity. A particular attention should be paid to the trawl codend which is the part of the trawl where fish are retained. Indeed, the codend hydrodynamics have as a non-negligible impact on its selection [1]. For instance, the codend fluttering motions lead to some changes in the codend mesh openings $[2,3,4]$ and may have direct consequences on cleaner catches, knowing that the catch is correlated to the codend behavior [5, 6, 7].

The analysis of the high Reynolds turbulent flow around a moving codend remains a challenge for both experimental and numerical approaches. Indeed, the immersion of such a fishing net structure in a turbulent flow induces structure oscillations that are directly linked to elastic, kinematic and dynamic instabilities. The origins of such structure oscillations for an immersed body in a turbulent flow are multiple [8]. The most common ones in the present flow configuration are i) induced vibrations caused by local hydrodynamic effects (fluctuating velocities, vortex shedding wake, etc); ii) induced vibrations caused by the warp tension variations, iii) induced vibrations due to movements of the deformable structure itself. Furthermore, the geometry of the rigid structure has a significant effect on the wake [9]. Therefore, in the current moving codend fishing net structure, some other potential sources of structure excitation are also present. They are related to the possible fluid-passage though the porous structure and also to the fully unsteady Turbulent Boundary Layer (TBL) flow developing inside and outside the fishing net surface $[10,3]$. Finally, every excitation mechanism may occur simultaneously and interacts with each other, increasing the complexity of the fluid-structure interaction characterization. As a consequence, some dimensionless parameters classically used for the Vortex Induced Vibration (VIV) analyses can not be used in the present flow configuration. For example, parameters such as dimensionless time and normalized oscillation amplitude (both related to body oscillations) or mass ratio can not be properly estimated here due to the unsteady character of the fluttering motion of the codend as well as to the instantaneous variability of the fishing net mesh opening.

Even if the flow around freely moving structures is of relevance for many practical applications, the complex flow interaction with a freely moving deformable structure remains poorly understood. In the past, some canonical flow configurations such as the wake of a rigid fixed cylinder or sphere have been fully investigated. The wake flow developing behind a rigid body which is forced to oscillate has been previously analyzed as well, demonstrating the classical lock-in phenomenon $[11,12]$. The dynamics of the flexible cable for which vortex induced vibrations in two degrees of freedom are present, have also been studied [13, 14]. These last studies and references therein may be related to present flow configuration even if bottom trawl can not be really identified as a flexible cable due to its particularities : flexible, porous and deformable. Besides, we must not forget that the body of the trawl is funnel-like, wide at its entrance and narrowing towards the codend. Even if the codend is of greater diameter than the cylindrical middle part of the trawl (see figure 1 - right hand side), trawl gear may be also related to an elongated body. However, the great disparities compared to previous studies dealing with elongated body concerns the flow which develops not only around the fishing structure but also inside it.

So far, there have been very few studies about the investigation of a freely moving codend structure and its fluid-structure oscillation interaction. Pichot et al. [15] previously used Laser Doppler Velocimetry (LDV) measurements to study the mean flow field over a rigid fixed codend with a closed and an open net entrance. Kim [16] performed one-point measurement inside a codend demonstrating the effect of turbulence on fish behavior. Based on Particle Image Velocimetry (PIV) measurements, Bouhoubeiny et al. [17] investigated the flow around a rigid codend and also around an oscillating codend model. These preliminary analyses showed the possible 
turbulent flow interactions with the fluttering codend structure without any full investigation of PIV database. In order to increase juvenile fish escape, Kim et al. $[18,19]$ recently proposed to implement an Active Stimulating Device (ASD) allowing to drive fish near the codend. Such an ASD technique induces fishing gear fluttering motions with varying amplitudes during fishing operations. Therefore, prior to implementing such a stimulating device, it seems important to determine the codend hydrodynamics and its associated oscillations frequency content. Moreover, a great disparity is obtained in the measurements when dealing with different flow conditions not only during real fishing operations [16] but also in a laboratory [20]. In this study, we propose a preliminary specific flow configuration which has been scaled to approach the real flow field around full scale codend. The analysis of the hydrodynamics and associated codend oscillations is then performed providing significant information that could be extrapolated to a full scale codend in certain conditions. More precisely, we focus on hydrodynamics behavior of rear codend even if the flow characteristics inside the codend may certainly also induce coded oscillations. However, it seems that the codend motions are mainly related to flow characteristics around the body. The codend movement is mainly linked to the lock-in phenomenon due to the vortex street developing behind the codend.

The present research is motivated by the lack of detailed quantitative information on the coupled dynamics of the codend fluttering motion and its hydrodynamic behavior. The aim of this study is to characterize the flow in the wake of a moving codend and to link the flow characteristics to the motion of the codend. We then propose to experimentally investigate the moving codend hydrodynamics of a 1/10 scaled bottom trawl. More specifically based on PIV measurements of the unsteady wake developing behind the moving codend structure, the frequency content of codend oscillations is characterized and linked to the physical mechanism related to the whole oscillating process.

\section{Experimental set up}

Experimental measurements are performed in the IFREMER (French Research Institute for Exploitation of the Sea) wave and current circulation flume tank whose dimensions are the following : $18 \mathrm{~m}$ (length) $\times 4 \mathrm{~m}$ (width) $\times 2 \mathrm{~m}$ (depth) $[15,17]$. The bottom of the flume is a conveyor belt which can be synchronized with the water speed in order to simulate devices in contact with the bottom, like bottom trawls. A side observation window of $8 \times 2 \mathrm{~m}^{2}$ allows users to observe the behaviour of the trawl models during trials and to carry out measurements. In this work, the hydrodynamics of a freely moving modeled bottom trawl are investigated with PIV measurements. The measurements are made with a constant streamwise velocity of $U_{\text {ref }}=0.4 \mathrm{~m} \cdot \mathrm{s}^{-1}$ and a turbulence intensity rate of $5 \%$. This turbulence level corresponds to the one computed in another far field measurement plane where the flow is uniform and based on the same inflow conditions. Using Reynolds decomposition, the turbulence level has been determined and it is of an order of $4.8 \%$. The input streamwise velocity corresponds to a towing speed of $2.5 \mathrm{knots}$ at real scale.

\subsection{Description of the $1: 10$ scaled codend model}

A $1: 10$ scaled model of a bottom trawl is considered in this work. Such a model is $4 \mathrm{~m}$ long with a swept width of $1.8 \mathrm{~m}$ (horizontal opening) and a swept height of $0.4 \mathrm{~m}$ (vertical opening). The rigging is composed of four bridles of $2 \mathrm{~m}$ long (with a diameter of $1 \mathrm{~mm}$ ) connected to $1 / 10$ trawl doors. The top bridles are fixed to the warp as shown in [6].

The fishing net structure is a $1: 10$ scaled model of a commercial two faces bottom trawl. The 

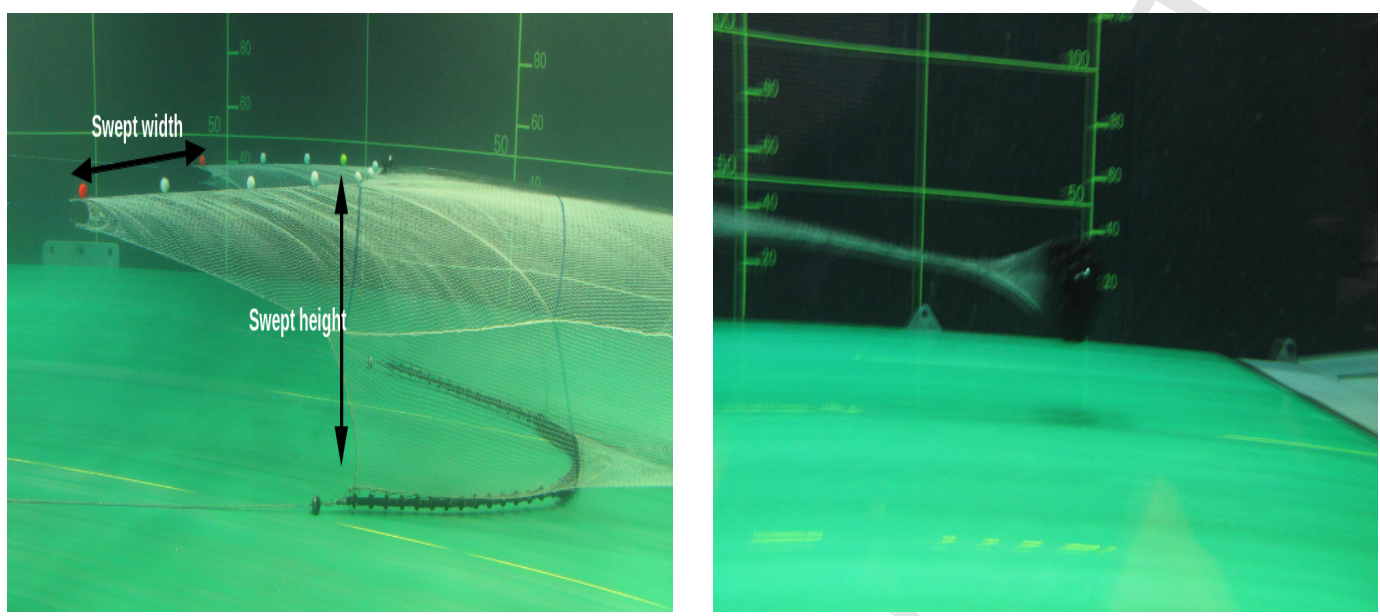

F $\quad 1: 1: 10$ scaled model of a bottom trawl. Entrance (left hand side) and moving codend (right hand side).

model is made with diamond-shaped meshes of $8 \mathrm{~mm}$ long (the length of the meshes between two knots), with a twine diameter of $0.6 \mathrm{~mm}$ [10]. 60 meshes have been used for the codend part, with a trawl design presented in [6].

Figure 1 displays the upstream part of the bottom trawl and its associated codend. The codend catch is simulated using porous balls. During experiments, no displacement of the catch are observed. The diameter of the codend at rest is $d=0.22 \mathrm{~m}$ (see figure 2 for the definition of $d$ ). The Reynolds number of the present flow configuration based on the flow velocity $U_{r e f}$ is $R e=U_{r e f} d / v=88000$, with $v$ the kinematic viscosity of water.

Note that an investigation of the Turbulent Boundary Layer (TBL) developing over the horizontal part of this bottom trawl has been already performed [10] demonstrating the influence of trawl motion onto the TBL flow characteristics. This preliminary study confirmed the great complex flow structure interaction mechanism in such a flow configuration.

\subsection{PIV measurements}

In this study, instantaneous velocity vector fields are measured in the symmetric plane of the codend with classical PIV apparatus (see figure 2). Glass spheres with a mean diameter of $15 \mu \mathrm{m}$ are used to seed the flow. The PIV method relies then on the determination of the motion of seeded particles by analyzing the displacements of particles between two successive images [21]. The interest of PIV is that measurements provide not only the spatial instantaneous description of flow structures but also their dynamics (time-frequency description).

The PIV system consists of a double pulsed laser type two-chamber Gemini PIV Nid-Yag $2 \times$ $120 \mathrm{~mJ}$ at $4 \mathrm{~Hz}$, and a Dantec Hi-sense CCD camera, with a focal lens length of $60 \mathrm{~mm}$. The laser is placed behind the codend and the camera is outside the tank. The camera has a $4 \mathrm{~Hz}$ frequency for a double-frame images with a $1600 \times 1200$ pixels $^{2}$ resolution. Finally, instantaneous velocity fields are obtained using a cross-correlation PIV algorithm. The size of the interrogation window is $32 \times 32$ pixels $^{2}$ and adjacent windows are $25 \%$ overlapped. Instantaneous velocity fields are then measured on the physical mesh of $n_{x} \times n_{y}=99 \times 74=7326$ points.

To investigate the near wake behind the codend net structure, the PIV system is implemented to determine the streamwise $u$ and vertical $v$ velocity components along the horizontal $x$ and 
$y / d$
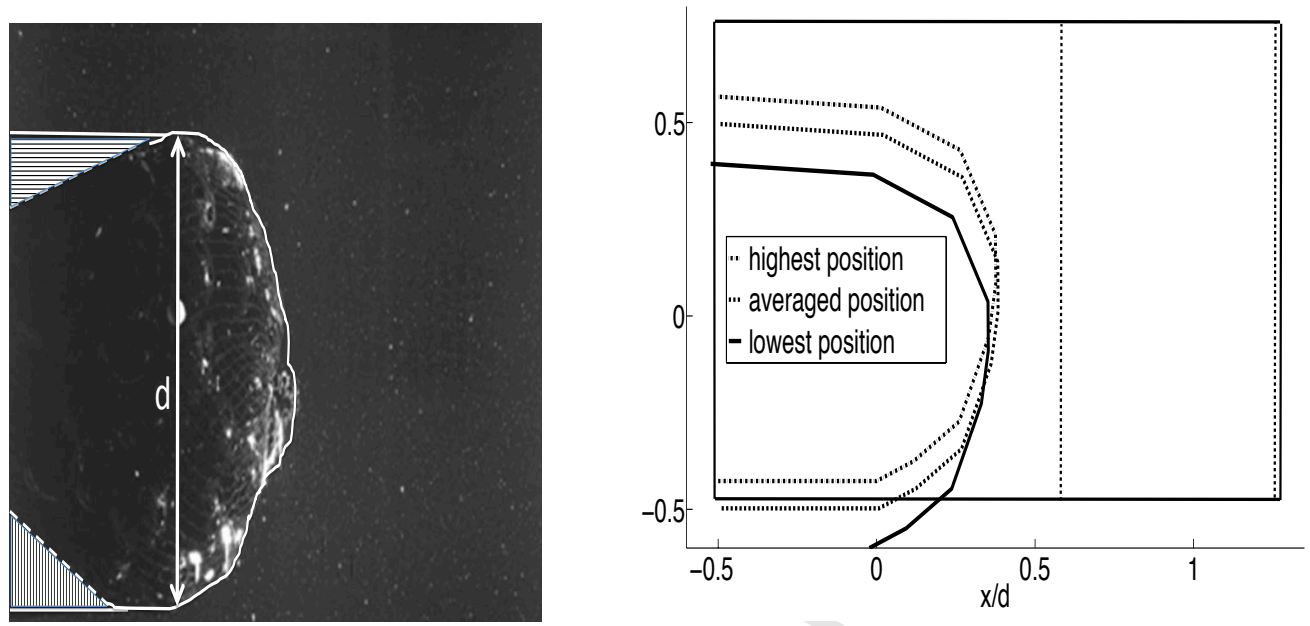

F 2: Left : Illustration of a Raw PIV image. Hatched white areas indicate the left part of the codend structure which can not be identified thanks to PIV image due to shadow effects. Right : Sketch of the PIV investigation area around the oscillating codend structure surface. PIV measurements plane included the highest and lowest positions of the codend during measurements. The two dashed vertical lines indicate the locations along with spectral analyses will be conducted (see $\$ 3.3$ and figure 7).

vertical $y$ directions respectively. An illustration of a raw PIV image is given in figure 2. Note that the PIV system can not enable us to evaluate the particle image displacement in the shadow area produced by the codend structure. This shadow area is located between the dashed white line and the white line given in figure 2. The white line indicates the contour of the structure around which PIV measurements are available. 814 instantaneous PIV velocity vector fields are then obtained on the regular mesh of 7326 points with a spatial resolution of $3.8 \mathrm{~mm}$ in both directions. The time step between two PIV vector fields is $0.25 \mathrm{~s}$.

\section{Results}

An analysis of the mean flow properties is first performed. Then, the codend fluttering motion is investigated by extracting the codend contour-line in each PIV image. It is to be noted that no fluttering of the wing net, of the float line or even the catch were observed. Finally, codend hydrodynamics are studied with an emphasis on the coupling between fluttering structure motions and codend hydrodynamics.

\subsection{Analysis of the mean flow properties}

Figure 3 presents two instantaneous planar velocity fields at two characteristic times corresponding to the extreme vertical positions of the codend structure during the measurements : i) the lowest one - left hand side and ii) the highest one - right hand side. The turbulent wake flow is clearly evidenced and is also greatly function of the structure displacement. As stated previously, one can not really appreciate the flow developing all around the codend structure, especially in the upstream part of the codend because of the shadow effect in the image. Indeed, the codend 
structure is axi-symmetric and close to a spherical-shape structure (see figures 1 and 2). In these figures, the shear layer vortices are observed. These vortices are clearly discernible (black circles) along the line $y / d=0.4$ (figure 2-left) and even for $x / d \approx 0.6$. They appear just after the codend structure indicating that the transition point moves upstream due to the codend oscillations.

Prior to discussing any mean flow properties, an analysis of the statistical convergence of the mean velocities and the turbulent stresses is conducted. It is known that the number of PIV samples is a compromise between processing capabilities and acceptable convergence of statistics $[22,23]$. For present convergence analysis, we extract velocity signals at selected locations in the measurement plane corresponding to high and low shearing areas. Five points (labelled $X_{i}$ with $\mathrm{i}$ varying from 1 to 5) are considered and they are indicated with black squares in a graph of the figure 5. Figure 4 displays the variation of averaged flow quantities as a function of the number of samples used to compute the statistics. Even if the time duration is certainly too small for properly investigating the very low frequency content of the velocity signals, these results demonstrate that a converged state is reasonably achieved. Present PIV statistics and spectral analysis presented in $\S 3.3$, would need longer time histories. It is then a first step towards a more detailed analysis.

Figure 5 represents the topology of the mean flow field computed in the context of the Reynolds decomposition. In each graph, the highest and lowest positions of the structure are indicated with a dotted-line and a continuous line respectively. Note that the unsteady motion of the structure avoids any use of the Reynolds decomposition in the area swept by the codend oscillations. That justifies zero values imposed in this domain. The mean flow pattern in the wake recirculation is classically dominated by two-symmetric vortices and it is quite similar to the one obtained behind a fixed rigid codend [17]. However, when comparing the mean wake flow topology of the present fluttering codend to a fixed codend [17], the locations of both symmetric vortex centers are slightly different. For the fixed rigid codend, the centers are approximately located at $y= \pm 0.5 d$ while in the present flow configuration, the associated centers are located at $y= \pm 0.35 d$. This is directly linked to the oscillating structure motion which tends to limit the transverse extent of symmetric vortices. On the other hand, the streamwise extent of the wake recirculation is higher (approximately $\approx 1.5 d$ ) than the one obtained behind a rigid fixed codend [17] and the one classically observed when dealing with the wake of a cylinder [24] or sphere obstacle. The recirculation length also depends on the structure's oscillation response. The length denoted $D$ (see figure 5) corresponding to the vertical spatial extent of the structure's $y$-oscillations is then estimated and $D$ is equal to $0.258 \mathrm{~m}$. This results in a recirculation length of $1.28 \mathrm{D}$ approaching classical previous observations. The fluttering motion of the codend structure in a fluid flow increases then the strength of the shed vortices that modifies the resulting drag force accordingly.

Figure 5 displays the following Reynolds tensor components : $\overline{u^{\prime 2}}, \overline{v^{\prime 2}}, \overline{u^{\prime} v^{\prime}}$, where an overbar indicates the time average. Results indicate that the Reynolds stresses are symmetric about the structure center axis. The center-axis is defined as the average $y$-center of the structure motion. The normal components are larger than the shear component. Such isosurface representations exhibit classical behavior $[17,24]$ even if the PIV measurement plane is limited to $x / d=1.3$ (that is $x / D=1.1$ ), avoiding any full investigation of the wake flow. The $\overline{u^{\prime 2}}$ component has a two-lobe structure taking its origin in the structure oscillation area. This suggests an increased unsteady activity in this area related to the structure's $y$-oscillations. The $\overline{v^{\prime 2}}$ component seems to have a one-lobe structure located outside the PIV measurement plane. The shear component $\overline{u^{\prime} v^{\prime}}$ exhibits two areas with opposite signs, located on either side of the wake centre line. 
$y / d$

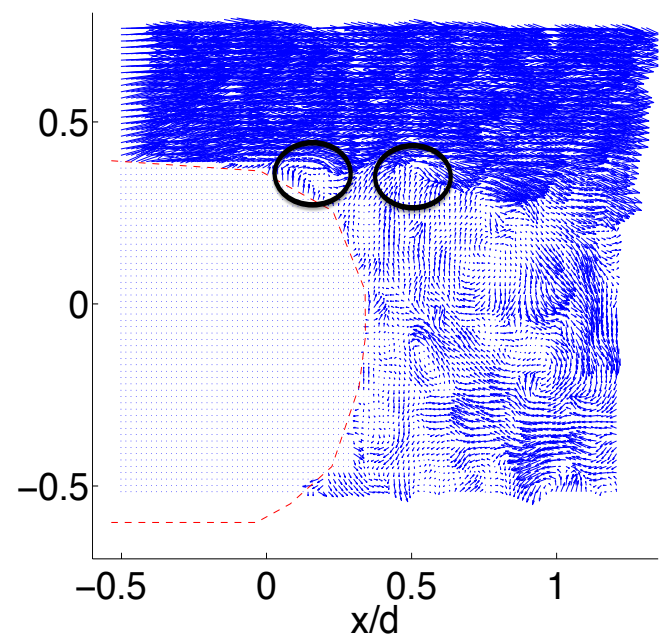

$y / d$

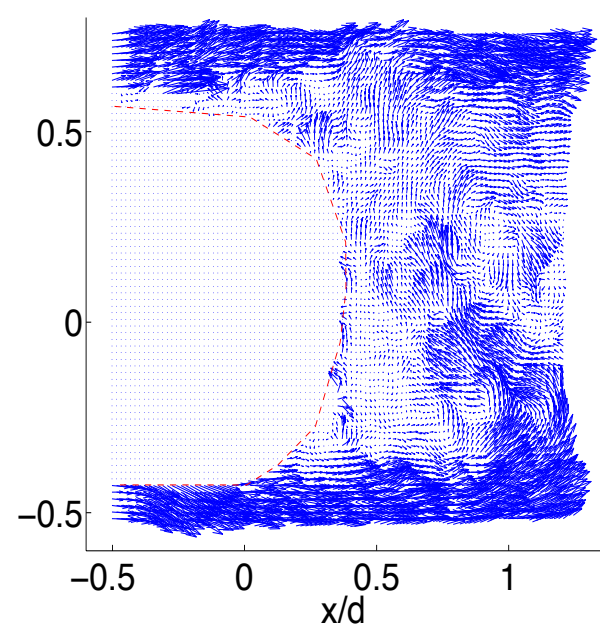

F 3: Illustration of two instantaneous PIV vectors fields. Left : lowest position of the codend structure. Right highest position of the codend structure.

Globally the topology of the mean flow field is close to classical results if one considers the vertical extent of the structure's oscillations as a reference length scale. Moreover, as previously observed for a vibrating cylinder [12], the wake flow pattern greatly depends on the structure's oscillations which are analyzed in the next section.

\subsection{Analysis of the codend fluttering motion}

The detection of the codend interface is implemented in each PIV image (figure 2) and consists of the following steps : nine points located on the surface of the bottom trawl are manually marked in each PIV picture. The fishing structure moves quite slowly, and the local fishing net deformation is quite small compared to the amplitude of the fishing structure oscillations. Thus, the time evolution of each marked point is similar in the PIV plane. That enables the time evolution of the whole fishing net structure to be determined during experiments.

Figure 6 displays the time evolution of the vertical and longitudinal oscillations of the codend structure. In this graph, the fluctuating parts of both signals are plotted. The structure's $y$-oscillation amplitude is more than twice the height of the one related to the $x$-oscillation. The peak to peak vibration amplitudes of $x$ - and $y$-oscillations are $0.057 d$ and $0.1735 d$ respectively that corresponds to $\pm 3 \%$ and $\pm 8.7 \%$ of $d$. The standard deviation of the $y$-displacement signal is also a little higher than the one of the $x$-displacement signal. In this graph, one also plots the $x-$ and $y$ oscillatory associated velocity. The maximum oscillatory velocity is $u_{x m}=0.019 \mathrm{~m} . \mathrm{s}^{-1}$ and $u_{y m}=0.064 \mathrm{~m} . \mathrm{s}^{-1}$ for $x-$ and $y$ - oscillations respectively. These values correspond to $5 \%$ and $16 \%$ of the reference flow velocity $U_{\text {ref }}$. Therefore, such a motion has a non-negligible effect on the codend hydrodynamics. The effect of codend's oscillations is to increase the resultant velocity of the fishing structure and, consequently, modify the codend hydrodynamics then the fluid forces and the mesh opening as the node positions will certainly change at any time. 
$\overline{u\left(X_{i}\right)}$

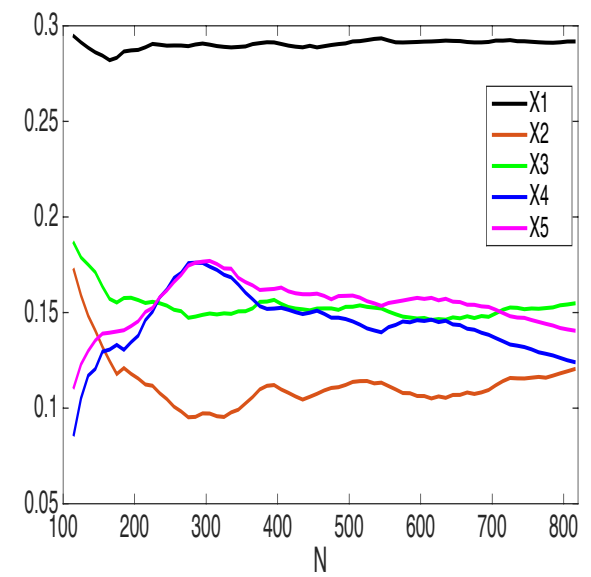

$\overline{u^{\prime 2}\left(X_{i}\right)} / U_{r e f}^{2}$

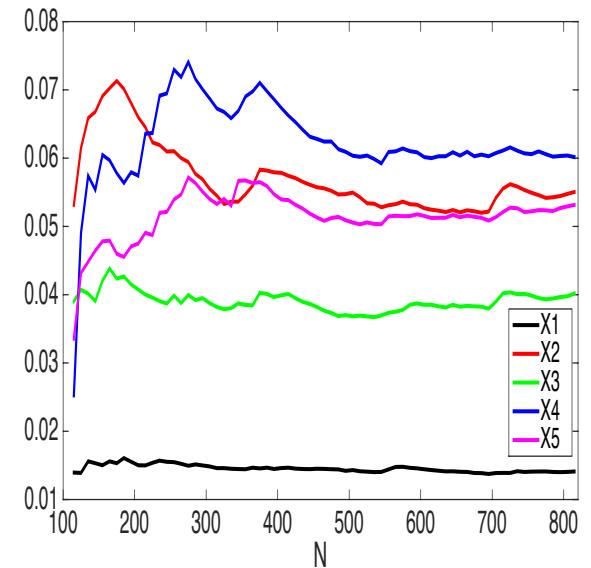

$\overline{u^{\prime}\left(X_{i}\right) v^{\prime}\left(X_{i}\right)} / U_{r e f}^{2}$

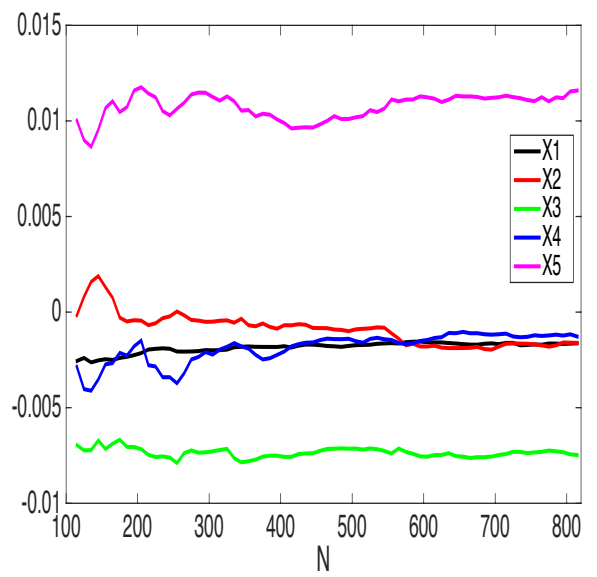

$\overline{v\left(X_{i}\right)}$

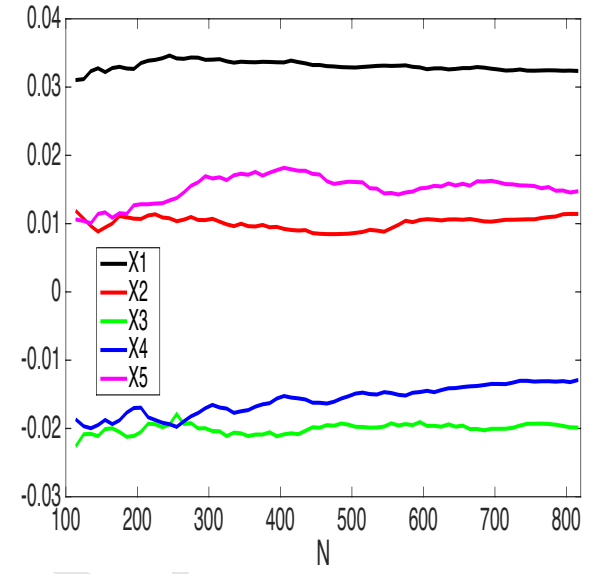

$\overline{v^{\prime 2}\left(X_{i}\right)} / U_{r e f}^{2}$

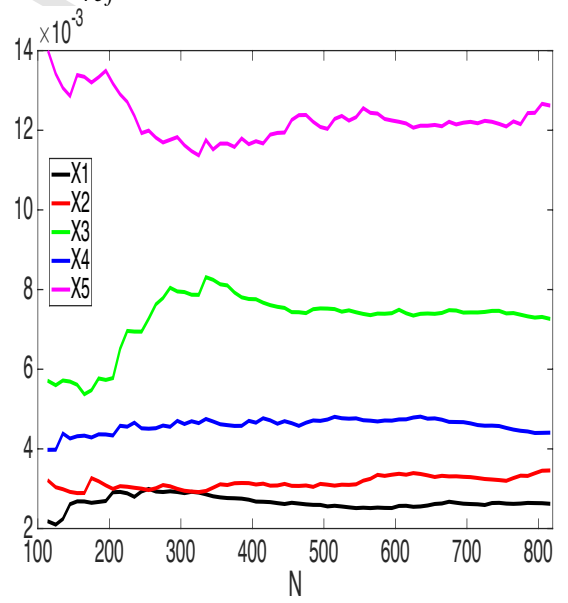

F 4: Convergence analysis of averaged flow quantities computed from $N$ consecutive snapshots. First line : Mean flow. Streamwise velocity component (left); transverse velocity component (right). Center line : Reynolds stresses. $\overline{u^{\prime 2}\left(X_{i}\right)} / U_{r e f}^{2}($ left $) ; \overline{v^{\prime 2}\left(X_{i}\right)} / U_{r e f}^{2}$ (right); bottom-left $: \overline{u^{\prime}\left(X_{i}\right) v^{\prime}\left(X_{i}\right)} / U_{r e f}^{2}$. 
$y / d$

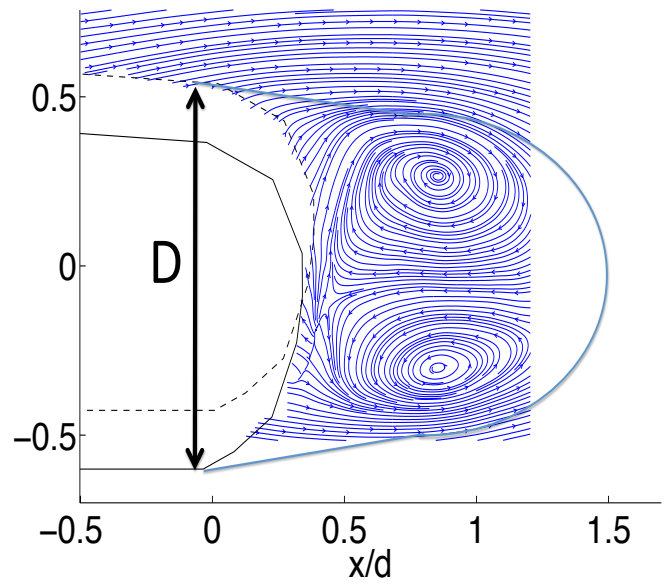
$y / d$

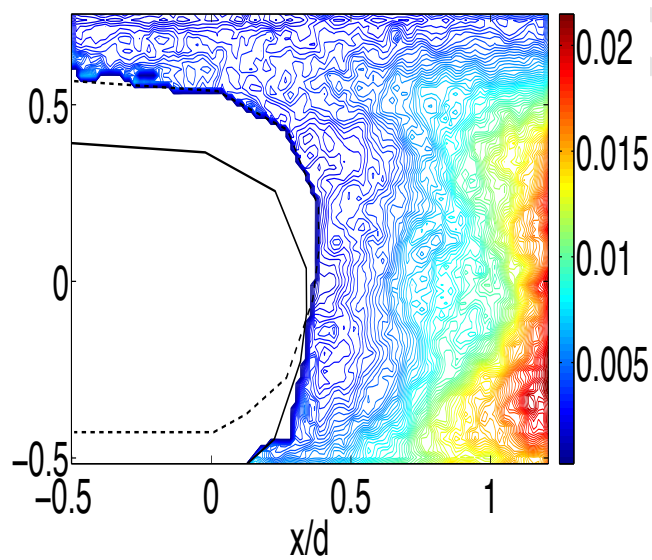

$y / d$

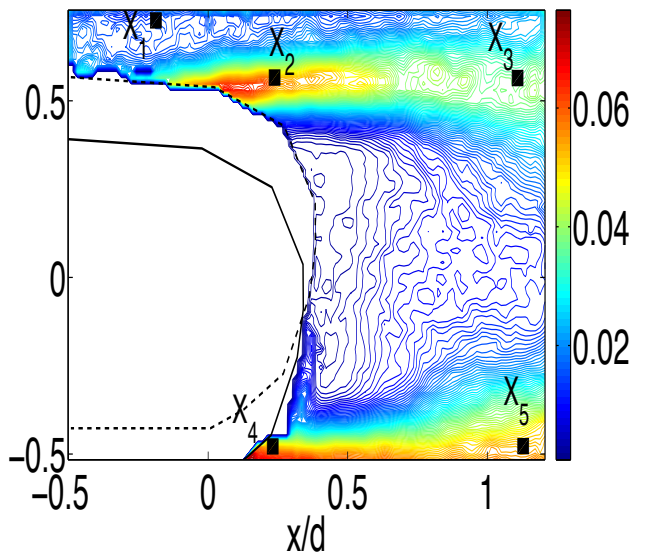

$y / d$

$\mathrm{F} \quad$ 5: Top-right : Mean streamline of the velocity field. Reynolds tensor components : $\overline{u^{\prime 2}}$ (Top-left) $\overline{v^{\prime 2}}$ (Bottom-right) $\overline{u^{\prime} v^{\prime}}$ (Bottom-left).

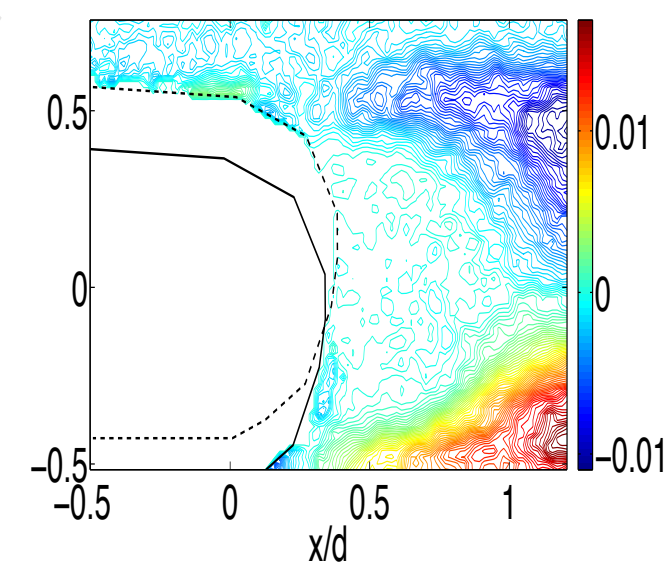


Regarding the discussed Fourier analysis in the following, given the complexity of the interaction, much longer signals should certainly be needed in order to evaluate the predominant frequency peaks with sufficient precision. In this sense, to improve the statistical convergence, the Welch method is retained to compute spectra. Each spectrum presented in the following figures is then computed using FFT based on Welch's method and using a Hann window.

In figure 6, the spectral representation of structure's oscillations is given in a log-log scale. The highest frequency peak is obtained at a very low frequency component $\left(f_{1}=0.01 \mathrm{~Hz}\right)$ for both $x$ - and $y$ - displacements. The second frequency peak is observed at $f_{2}=0.21 \mathrm{~Hz}$, especially for the transverse $y$-displacement. Note that the interactions between the low frequency component $\left(f_{1}\right)$ and the $f_{2}$ frequency induce an additional frequency $f_{3}=f_{2} \pm f_{1}$, especially observable for $f_{3}=f_{2}-f_{1}=0.20 \mathrm{~Hz}$. Globally, the codend's oscillations are fully unsteady and the spectrum of the structure' $x$-oscillations exhibits mainly a frequency peak around $f_{1}=0.01 \mathrm{~Hz}$ while the spectrum of the structure's $y$-oscillations exhibits a more broadband spectrum with two pronounced peaks around $f_{1}$ and $f_{2}$ frequencies.

Note that low frequency of the codend is independent on frequency of the incoming flow. Indeed, previous velocity measurements were performed in the same flume tank however based on different flow configurations (for instance, flow around a rigid codend [17] or marine current turbine flow [25]) and the velocity spectra did not exhibit such a low frequency content.

Based on these frequency values, the dimensionless Strouhal number $S_{t}=f L / U$ with $\mathrm{f}$ the frequency oscillation, $L$ the reference length and $U$ the reference velocity is calculated. One obtains $S_{t 1}=f_{1} D / U_{\text {ref }}=0.0065$ and $S_{t 2}=f_{2} D / U_{\text {ref }}=0.135$. Then the second frequency peak $\left(f_{2}\right)$ is related to the vortex shedding frequency of the natural wake flow excitation. Indeed, as it has already been demonstrated, the vortex shedding frequency of a structure in motion is smaller than the vortex shedding frequency of a structure at rest. For a similar flow configuration but with a rigid fixed codend, Bouhoubeiny et al. [17] showed that the Strouhal number related to the vortex shedding frequency was 0.19 . Conversely, the low frequency motion seems to be directly linked to the cyclic variations in the warp tension related also to the elastic nature of the elongated bottom trawl. This is reinforced with the fact that the $f_{1}$ frequency is the main frequency detected in the structure $x$-oscillation. This low-frequency appears as the only component in the spectra of transverse and streamwise oscillations, indicating that these oscillations are locked into the same kind of dynamics which is certainly related to the warp tension enabling the bottom trawl to be maintained horizontally. Furthermore, this low frequency component is quite similar to the one also detected on the horizontal surface of this bottom trawl [10]. This result is also coherent with previous measurements of cable forces [26] enabling a fluttering sheet of net to be maintained in a flow [3]. Indeed, these measurements have shown that the cable force signal is of low frequency content [26].

Finally, the codend structure in a fluid flow will vibrate due to the regular shedding of vortices into the wake and also to the warp tension maintaining the codend horizontally. The predicted amplitude of codend oscillations depends upon the difference between the frequencies associated with both phenomena and the oscillations due to the warp tension are the most dominant ones. This result is quite comparable to previous work dealing with VIV of a long flexible structure in cross-flow [14]. They observed that the in-line direction oscillations dominated the cross-flow ones and these in-line oscillations arised from the tension stiffness. Note that it is quite interesting to observe that the computation of the Strouhal number based on the $f_{1}$ and on the streamwise velocity of the $x$-oscillation : $S_{t 1^{\prime}}=f_{1} \times D / u_{x m}$ provides $S_{t 1^{\prime}}=0.135$. This is close to the value of $S_{t 2}$ previously computed and related to the vortex street. This result emphasizes the great coupling that exists between the vortex sheet and the low frequency fluttering motion of the 
structure.

\subsection{Fourier analysis of PIV velocity field}

The spectral content of the instantaneous velocity fields extracted along two vertical lines is now studied. More precisely, the streamwise and vertical velocity components are stored at two $y$ lines located just after the codend $(x / d=0.58)$ and in the wake flow $(x / d=1.27)$. These vertical lines are indicated in figure 2 with dotted lines. Figure 7 represents velocity spectra represented as a function of $y$-positions ( $x$-axis) and frequencies ( $y$-axis). These spectra are normalized with the maximum value of these four spectra. Note that this maximum is nearly the same for the spectra of the streamwise velocity component along both $y$-lines but for the $y$-line $(x / d=0.58)$ this maximum is attained for $y / d= \pm 0.5$ at low frequencies $\left(f \approx f_{1}\right)$ while for the other $y$ line $(x / d=1.27)$ this maximum is attained for $y / d \in \pm[0.4: 0.55]$ at frequency $f=f_{2}$. When regarding raw spectra, the amplitude of transverse velocity spectra is more than ten times smaller than that of streamwise velocity spectra. This is mainly due to the difference between streamwise and transverse velocity component values.

Firstly, the $u$ spectra exhibit both $f_{1}$ and $f_{2}$ frequencies in the shear layer of the wake flow. Such shear layer extends along the vertical direction and covers the length swept by the structure's $y$-oscillations. In the separating shear layer $(x / d=0.58)$, one also observes the subharmonics of the main low frequency component $\left(f_{1}\right)$ of the codend oscillations. The streamwise velocity component is very sensitive to the low frequency oscillations. It is then indicated that the main flow instabilities in the shear layer originate from the codend motion.

Secondly, the transverse velocity component is mainly related to the vortex shedding frequency $\left(f_{2}\right)$ in each vertical line with an emphasis in the wake $(x / d=1.27)$. These results confirm the lock-in phenomenon which is that the vortex shedding frequency coincides with the structure's oscillations at frequency $f_{2}$. However, it is interesting to note that in the developing wake $x / d=1.27$, the low-frequency component is still present at a smaller amplitude than the one associated with the $f_{2}$ frequency. The topology of the low-frequency activity is different from the vortex shedding street but it is still present in the wake indicating that this is a slow process, and a long-lived one. This confirms that both frequency peaks of the codend's oscillations are quite fully recovered in the wake of the codend.

Previous VIV analysis of the near wake instabilities of a rigid fixed cylinder at $R e=10000$ [27] have shown that the shear layer vortices were observed to occur at higher frequencies than the Strouhal vortices. Here, the frequency instability of shear layer vortices are directly dictated by the low-frequency codend motion. Codend hydrodynamics are then very sensitive to the structure's oscillations, and vice versa. Such results will certainly have some implications on the catchability. Indeed, as it has been demonstrated in Bouhoubeiny et al. [3], a similar fully unsteady TBL flow is developed both inside and outside the horizontal part of the fishing net structure. Then, it is expected that inside the codend, the codend fluttering motion induces some hydrodynamics having similar frequency content. As a direct application, the knowledge of the codend motion would certainly enhance the efficiency of Active Stimulating Device [18, 19] previously proposed to active fish stimulus escapement.

\subsection{Vortex shedding street analysis}

To pursue the analysis of the wake hydrodynamics, we propose to extract the vortex shedding street from PIV database. For such analysis, a Fourier filtering procedure is implemented. It consists in first performing a FFT of the available instantaneous vector PIV fields. Then in 

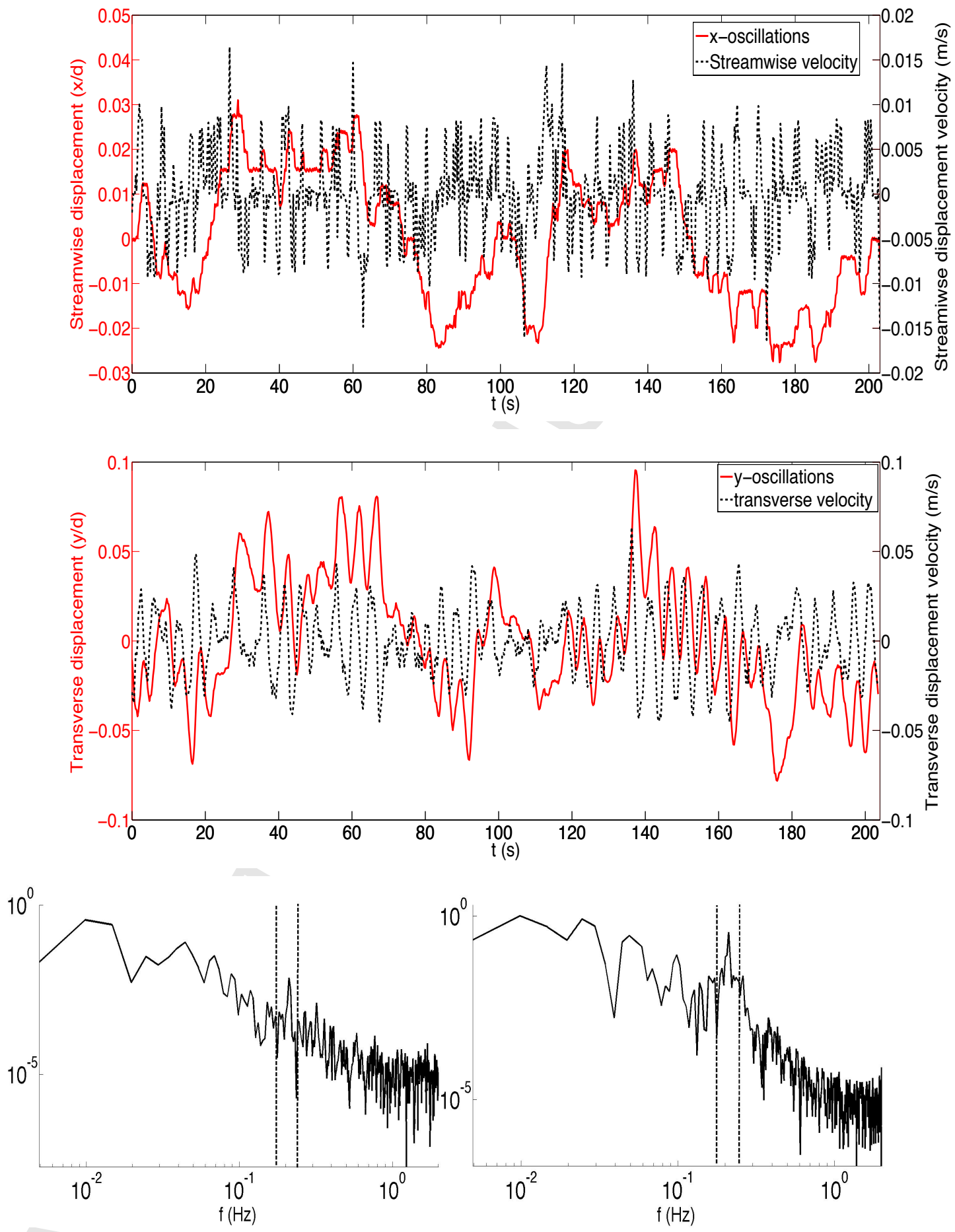

F 6: Top : Fluctuations of the $x$-structure oscillations normalized with the codend diameter $(d)$ and superimposed on the associated oscillatory $x$-velocity. Center : Fluctuations of the normalized $y$-structure oscillations superimposed on the associated oscillatory $y$-velocity. Bottom : Spectral representations (in a log-log scale) of both $x$ - (left) and $y$ - structure oscillations (right). 
1

2

3 $y / d$

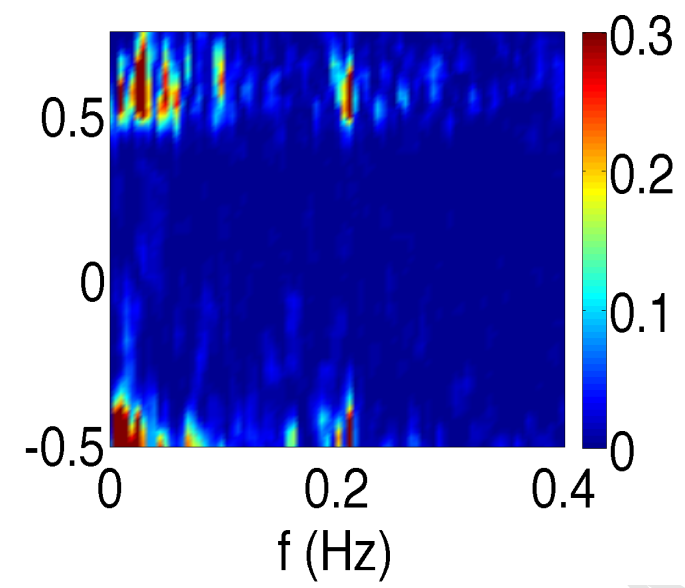

$y / d$

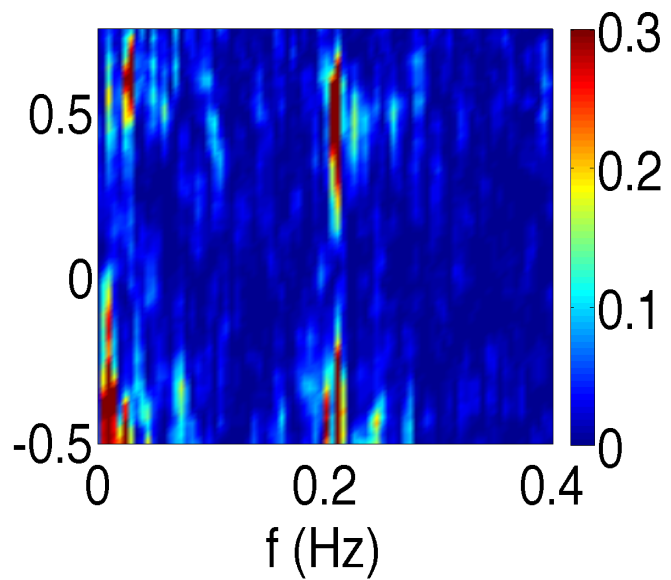

$y / d$

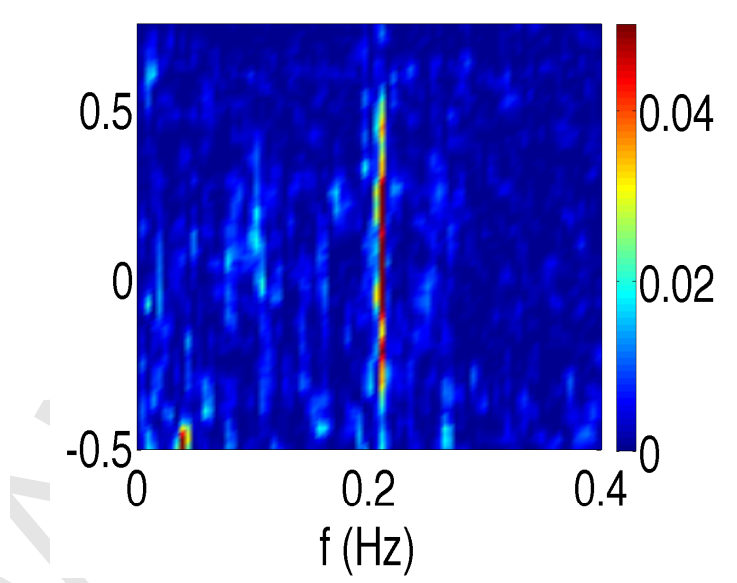

F 7: Spectral representation (linear scale) of the streamwise (left) and transverse (right) velocity components along the lines $x / d=0.58$ (top) and $x / d=1.27$ (bottom). Each spectrum is normalized with the same value.

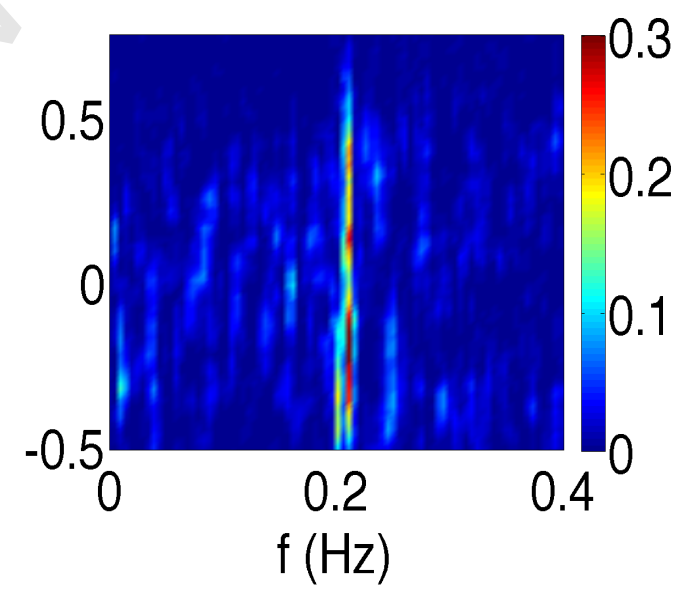


Fourier space, one only selects the frequency domain located around $f_{2}$ (see figure 6 where two dashed vertical lines delimit the domain). Then an inverse FFT is done to recover the velocity fields filtered at $f_{2}$ corresponding to the vortex shedding street. An illustration of such a filtering procedure is presented in figure 8 . It represents eight instantaneous filtered velocity streamlines from $t_{1}=154.5 \mathrm{~s}($ graph $(a))$ to $t_{2}=158 \mathrm{~s}($ graph $(\mathrm{h})$ ). The time interval between two consecutive images is equal to two times the time step of PIV measurements. Vertical lines in figure 6-center indicate the temporal domain under consideration that corresponds to approximately one cycle of $1 / f_{2}$ period of the movement of the codend structure. The vortex shedding street is then clearly visible on such graphs. Due to the limitation of the PIV measurement plane area, one only focuses in the following on the vortex generated and developed in the upper part of the codend. To appreciate the vortex street development, a specific algorithm previously developed [28] is applied to detect instantaneously the vortex core on each filtered vector field. Briefly, the vortex center has to fulfill three main criteria : (1) its neighboring instantaneous angular velocities have to be oriented in the same direction, (2) its neighboring angular velocity must have a higher absolute value than the center velocity and (3) the absolute velocity value for the vortex center has to be very low. This algorithm is then applied to each available filtered velocity field enabling the determination of the center coordinates of the vortex developing in the upper part of the wake. As an illustration, these centers are indicated with a black point in each graph of figure 8. Figure 9 (left) displays the temporal evolution of the $y$-coordinate (normalized with $d$ ) of the detected vortex centers. Red points indicate the envelop of this representation. It is quite interesting to observe that the spectrum of the envelop of these $y$-coordinates exhibits a low frequency component equal to $f_{1}$ frequency. This confirms that the vortex street is also linked to the low frequency codend's oscillations.

Figure 10 represents a zoom view of the time evolution of the vortex core $y$-motion during the same $1 / f_{2}$ period previously detailed. It is superimposed on the transverse motion of the codend (already represented in figure 6-center). We observe that the transverse motions of the vortex centers and of the codend are convected away in opposite directions. When the codend has an upward motion, the vortex initiated in the upper shear layer goes downward due to the local low pressure area located just behind the codend.

This result emphasizes the great complex non-linear coupling phenomena in the present fluidstructure interactions. Specific mathematical post-processing tools have to be applied to pursue this investigation allowing a characterization of the full understanding of the complex non-linear mechanisms in such a flow configuration. That would also enable to be fully examined the flow instabilities. However, one of the first difficulties of post-processing present database concerns the unsteady character of the moving interface. A solution may consist in using the phase averaged operator based on Proper Orthogonal Decomposition previously developed [10, 3]. Such analysis is ongoing.

\section{Conclusion}

PIV measurements was carried out to investigate the hydrodynamics around a fluttering $1 / 10$ scaled model codend in the symmetric plane of the structure. We first examined the mean flow properties of the wake developing behind the fluttering structure. It was observed that the mean flow characteristics greatly depend on the structure oscillations and strongly approach the mean flow characteristics of the wake developing behind a rigid body if one considers the vertical extent of the codend's oscillations as a reference length scale. The fluttering motion of the codend was then elucidated. We supposed that the main codend's oscillations are in the transverse and 

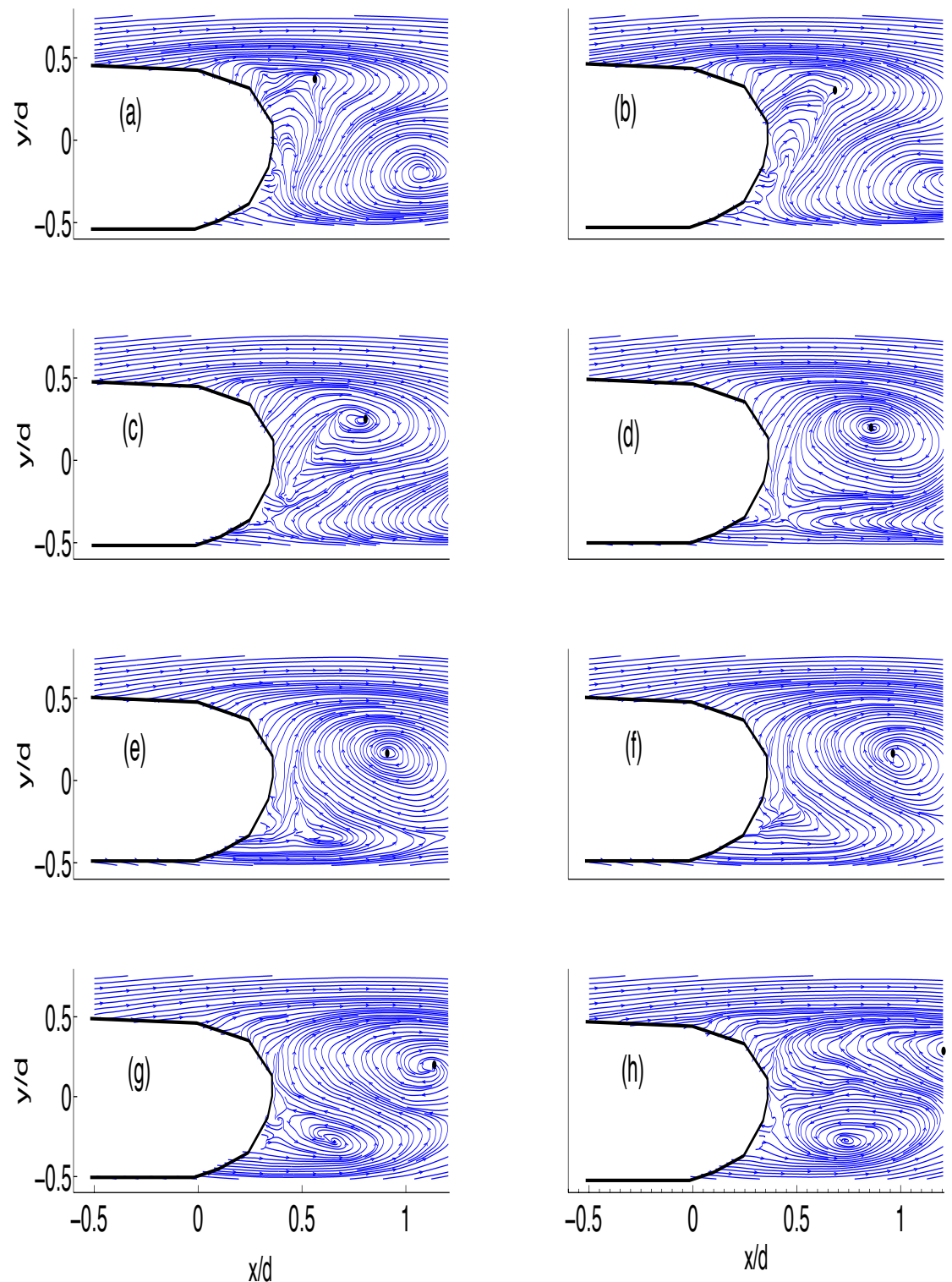

F 8: Illustration of a vortex core detection applied to filtered PIV velocity database. Representation of eight instantaneous filtered velocity streamlines (from $t_{1}=154.5 \mathrm{~s}\left(\right.$ graph (a)) to $t_{2}=158 \mathrm{~s}($ graph $(\mathrm{h})$ ). 
$y_{c} / d$

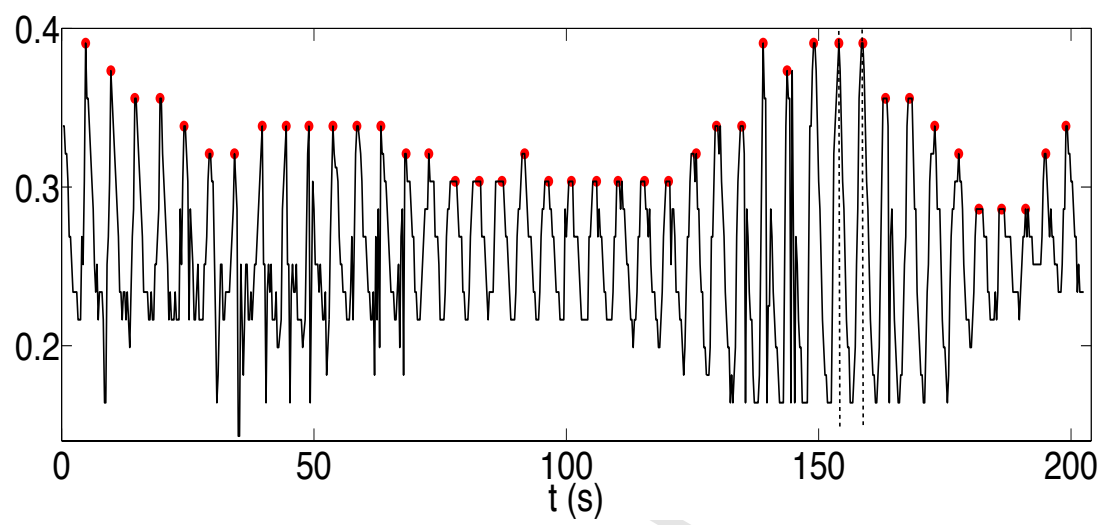

F 9: Time evolution of the transverse $y$-coordinate $\left(y_{c}\right)$ of the detected vortex cores.

$\underline{y_{c} / d, y / d}$

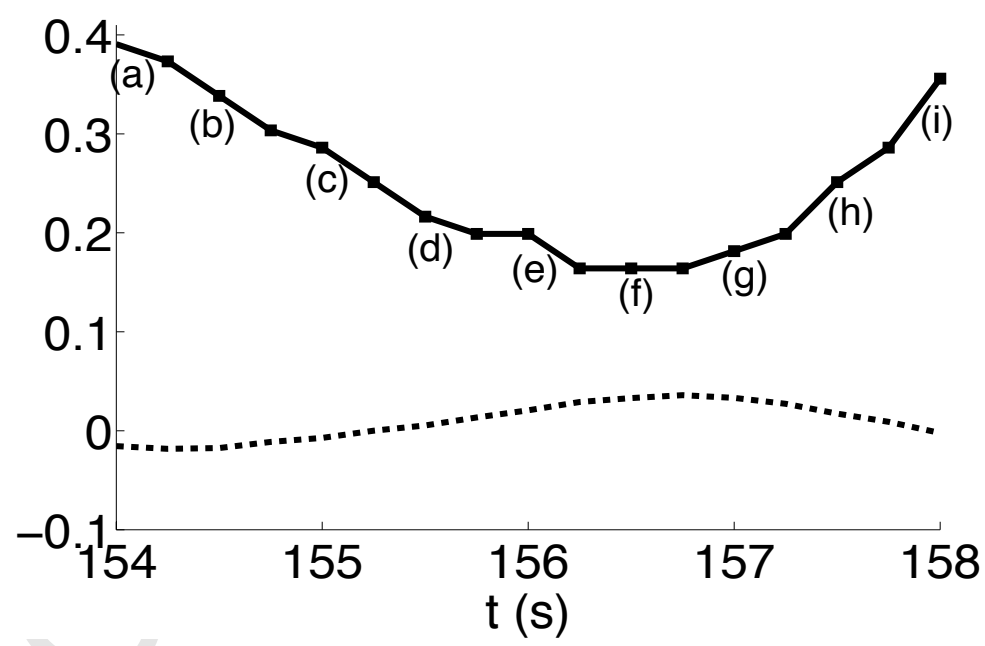

F 10: Superimposition of the $y$-displacement $\left(y_{c} / d\right)$ of the vortex core (line) and the transverse displacement $y / d$ (dashed line) of the structure. 
longitudinal directions. The motion of the codend is to be seen as a low-frequency component superimposed to another component associated with the vortex shedding street. The first one is directly related to the tension variation of the warp which enables the fishing structure to be maintained horizontally. The second one corresponds to the lock-in phenomenon related to the vortex street developing behind the fluttering structure. The topology of the low-frequency activity is different from the vortex shedding street. This fluttering motion changes the fluid force acting on the codend and also the local porosity of the codend. As measurements are performed only for a specific flow configuration with a $1 / 10$ scaled codend model, it is then expected that the amplitude of the codend oscillations may be 10 times higher during fishing operations, so a vertical motion of the order of a tenth of the codend diameter.

The periodic vortex shedding affects the codend motion. On the other hand, the oscillating structure will affect the fluid flow around the codend, the fluid force and the vortex pattern. Consequently, a complex fluid-structure interaction is present. The present study is a preliminary work demonstrating that the near wake hydrodynamics of moving codend structure are an intricate interplay between the codend fluttering motion and the shear layer instabilities and large scale vortex shedding. The non-linear flow instabilities require further investigations to elucidate the significant coupling between the fluttering motion and the hydrodynamic behavior. For such a purpose, specific post-treatment tools such as Proper Orthogonal Decomposition would be a solution to perform a phase averaging operation and to provide flow decomposition based on an energetic criterion. It is to be kept in mind that hydrodynamics of the codends might change with higher or lower catches. Present study is a preliminary experiment to reproduce the codend hydrodynamics and the associated codend fluttering motions. It allows the observations of the main flow characteristics developing around oscillating codend. This work also proposes a methodology for the analysis of flow characteristics and associated codend oscillations. It is then expected that present results and especially the codend movements provide significant information that could be extrapolated to a full scale coded under certain conditions.

The knowledge of such flow instabilities related to the codend fluttering motion is of great importance not only for structural models used to predict the selectivity process of the codend [29] but also to improve the understanding of the force acting on a trawl allowing the characterization of the shape, drag and behavior of the fishing structure during a trawling process, tension and loads in its threads and ropes. This also has a consequence on the fish moving process and then on the selectivity. Finally, some other parameters such as environmental conditions (sea state, ship motions, ...) have also a non-negligible influence on trawl performance [30].

\section{Références}

[1] F. O’Neill, S. McKay, J. Ward, A. Strickland, R. Kynoch, A. Zuur, An investigation of the relationship between sea state induced vessel motion and cod-end selection, Fisheries Research 60 (2003) 107-130.

[2] S. Gjosund, B. Enerhaug, Flow through nets and trawls of low porosity, Ocean Eng. 37 (2010) 345-354.

[3] E. Bouhoubeiny, P. Druault, G. Germain, Phase-averaged mean properties of turbulent flow developing around a fluttering sheet of net, Ocean Eng. 82 (2014) 160-168.

[4] P. Druault, G. Germain, J. Facq, PIV measurements combined with the motion tracking technique to analyze flow a around moving porous structure, J. Fluids Struct. 56 (2015) 190-204.

[5] N. Madsen, K. Hansen, N. Madsen, Behavior of different trawl codend concepts, Ocean Eng. 108 (2015) $571-577$.

[6] D. Priour, D. la Prada A., An experimental/numerical study of the catch weight influence on trawl behavior, Ocean Eng. 94 (2015) 94-102.

[7] H. Digre, U. Hanse, U. Erikson, Effect of trawling with traditional and 't90' trawl codends on fish size and on different quality parameters of cod gadus morhua and haddock melanogrammus aeglefinus, Fisheries Research 76 (2010) 549-559. 
[8] E. Naudascher, D. Rockwell, Flow-Induced Vibrations : An Engineering Guide, Courier Dover Publications, 2012.

[9] R. Blevins, Flow-Induced Vibration, Vol. 2nd New edition of Revised edition, Krieger Publishing Company, 2006.

[10] P. Druault, E. Bouhoubeiny, G. Germain, POD investigation of the unsteady turbulent boundary layer developing over porous moving flexible fishing net structure, Exp. Fluids 53 (1) (2012) 277-292.

[11] P. Bearman, Vortex shedding from oscillating bluff bodies, Ann. Rev. Fluid Mech. 16 (1984) 195-222.

[12] C. Williamson, R. Govardhan, Vortex induced vibrations, Ann. Rev. Fluid Mech. 36 (2004) 413-455.

[13] N. Jauvtis, C. Williamson, The effect of two degrees of freedom on vortex-induced vibration at low mass and damping,, J. Fluid Mech. 509 (2004) 23-62.

[14] Y. Modarres-Sadeghi, F. Chasparis, M. Triantafyllou, M. Tognarelli, P. Beynet, Chaotic response is a generic feature of vortex-induced vibrations of flexible risers, J. Sound Vib. 330 (2011) 2565-2579.

[15] G. Pichot, G. Germain, D. Priour, On the experimental study of the flow around a fishing net, Eur. J. Mech. B/Fluids (2009) 103-116

[16] Y. Kim, Analysis of the turbulent flow and tilt in the cod-end of a bottom trawl during fishing operations, Ocean Eng. 64 (2013) 100-108.

[17] E. Bouhoubeiny, G. Germain, P. Druault, Time-resolved PIV investigations of the flow field around rigid cod-end net structure, Fisheries Research 108 (2-3) (2011) 344-355.

[18] Y. Kim, D. Whang, An actively stimulating net panel and rope array inside a model cod-end to increase juvenile red seabream escapement, Fisheries Research 106 (2010) 71-75.

[19] Y. Kim, Fluttering characteristics of the ropes and nets as an active stimulating device inside the cod end of a trawl net, Fish. Aqua. Sc. 16 (2) (2013) 101-108.

[20] D. Priour, F. O’Neill, A. Sala, P. Chevaillier, B. Herrmann, Premecs-ii : Development of predictive model of codend selectivity, Q5rs-2002-01328, IFREMER (2006).

[21] R. Adrian, J. Westerweel, Particle image Velocimetry, Cambridge Aerospace Series, 2010.

[22] U. Ullum, J.J. Schmidt, P.S. Larsen, D.R. McCluskey, Statistical analysis and accuracy of PIV data, J. Visualization, 1(2) (1998) 205-216.

[23] O. Uzol, C. Camci, C. The effect of sample size, turbulence intensity and the velocity field in the experimental accuracy of ensemble averaged PIV measurement, in : 4th International Symposium on Particle Image Velocimetry, 2001.

[24] M. Braza, R. Perrin, Y. Hoarau, Turbulence properties in the cylinder wake at high reynolds numbers, J. Fluids Struct. 22 (2006) 757-771.

[25] O. Duran Medina, F. Schmitt, R. Califf, G. Germain, B. Gaurier, Correlation between synchronised power and flow measurements, a way to characterize turbulence effects on a marine current turbine, in : European Wave and Tidal Energy conference, 2015.

[26] J. Facq, T. Bacchetti, G. Germain, B. Gaurier, Compte rendu d'essai de mesures d'efforts et de géométries sur une nappe d'alèse (in french), Tech. rep., IFREMER (2010).

[27] S. Dong, G. Karniadakis, A. EkMekci, D. Rockwell, A combined direct numerical simulation-particle image velocimetry study of the turbulent near wake, J. Fluid Mech. 569 (2006) 185-207.

[28] P. Druault, P. Guibert, F. Alizon, Use of Proper Orthogonal Decomposition for time interpolation from PIV data, Exp. Fluids 39 (2005) 1009-1023.

[29] F. O’Neill, B. Herrmann, Presemo : predictive model of codend selectivity - a tool for fishery managers, ICES J. Marine Science 64 (8) (2007) 1558-1568.

[30] D. Queirolo, C. Hurtado, E. Gaete, M. Soriguer, K. . Erzini, C. Gutierrez-Estrada, Effects of environmental conditions and fishing operations on the performance of a bottom trawl, ICES J. Marine Science 69 (2) (2012) $293-302$. 